\title{
LIF/Mie Droplet Sizing of Water Sprays from SCR System Injector Using Structured Illumination
}

\author{
Łukasz Jan Kapusta \\ Warsaw University of Technology, Faculty of Power and Aeronautical Engineering, Institute \\ of Heat Engineering, Warsaw, Poland \\ Lukasz.Kapusta@itc.pw.edu.pl
}

\begin{abstract}
Recent trends in SCR (Selective Catalytic Reduction) systems development increase requirements for UWS (Urea Water Solution) injection. Close-coupled SCR system designs decrease the distance available for water evaporation and urea decomposition. Due to that, much effort is put into static mixing elements design improvement and injection process enhancement. So far, most experimental studies on UWS spray formation were based on Mie scattering visualization using global illumination and shadowgraphy imaging. High speed imaging of Mie signal with global illumination allows to determine global spray parameters such as penetration and angle but does not give information on droplet sizes. Droplet size determination, due to relatively large droplets generated by SCR injectors, can be done with Mie scattering or backlight imaging methods. Then the visualized area becomes narrowed since high magnification is required. Determination of droplet size distribution across whole spray in such arrangement requires number of measurements. LIF/Mie (Laser Induced Fluorescence/Mie scattering) technique provides an attractive alternative for rapid determination of droplet size distribution across the whole spray. This method however suffers from multiple scattering effects which might affect droplet size distribution results even in relatively dilute sprays.

In this study, LIF/Mie ratio distribution across sprays from commercial automotive injector for SCR systems was determined by simultaneous LIF and Mie detection using structured illumination. Moreover, the results were compared with conventional LIF/Mie imaging. Nd:YAG pulse laser was used as a light source. Second harmonic beam of $532 \mathrm{~nm}$ was used to illuminate the sprays. Instead of UWS pure water doped with Eosin $Y$ was used. The results showed that conventional images exhibited much stronger background signal. Moreover, the conventional imaging was sensitive to reflections from experimental setup elements, specifically reflections from LIF camera filter. These two observations prove the importance of using SLIPI for LIF/Mie droplets sizing in sprays for SCR systems. At the same time the obtained results showed that under certain conditions (no accidental reflections in the background) conventional imaging provides similar LIF/Mie ratio as structured illumination. The results showed that the LIF/Mie ratio remains unchanged over the spray cloud. This suggests that SMD remains unchanged as well. The slight increase of LIF/Mie ratio far from the injector outlet could be caused by absence of small droplets due to lower momentum and thus lower penetration distance. This assumption however should be verified with PIV measurement.
\end{abstract}

\section{Keywords}

SLIPI, LIF/Mie, droplet sizing, SCR, spray.

\section{Introduction}

The SCR aftertreatment concept for NOx removal from exhaust gases has over 10-year history in automotive sector. At the beginning, the SCR aftertreatment systems were implemented into heavy-duty vehicles [1]. Recently introduced NOx limitations [2] forced their application into passenger cars. As shown by Jaworski et al. [3] and Zheng et al. [4] previous aftertreatment system designs based on in-line arrangement of separate components such as oxidation catalyst, SCR catalyst, particulate filter, and ammonia trap allowed to place the injector relatively far upstream from the inlet of the SCR catalyst. The injector position could be easily optimized. The static mixer could be placed at distance of several duct diameters upstream the SCR catalyst as well. This allowed to provide sufficient distance for water evaporation and urea decomposition. Newly designed SCR systems are supposed to provide higher NOx reduction efficiency than in-line systems since the real driving conditions emissions measurement is supposed to be introduced in near future [5]. The stricter requirements for NOx emissions are the reason for close-coupled to the engine SCR systems designs. Such designs, due to higher exhaust gas temperature, offer huge potential of NOx reduction [6] but meet serious constraints in terms of packaging [7] and cause challenges for UWS injection. Integration of oxidation catalyst, SCR catalyst and particulate filter in one unit result in dramatically decreased space for water evaporation and urea decomposition. Therefore, each close-coupled SCR unit requires 
specific design for certain engine and vehicle. In many cases only one injector location is possible, which can be only slightly modified. Then, the injection process becomes of high importance since the spray pattern and initial droplet size distribution generated by the injector are the only factors which can be used to optimize spatial liquid particles distribution in the SCR system in terms of efficient liquid-gas mixing process. The spray pattern and droplet size distribution need to be adjusted to specific SCR unit design. Thus, fast and reliable method for spray characterization is very important for proper injector selection and spray pattern optimization.

Injectors for UWS injection which are used in the newest SCR systems provide relatively dilute sprays with high SMD (Sauter Mean Diameter), especially when compared to Diesel sprays. SMD in modern SCR system injector is typically around $100 \mu \mathrm{m}$ (measured at distance of $50 \mathrm{~mm}$ from the injector outlet). Thus, broad variety of measurement techniques can be used for droplet size determination starting from Mie scattering [8], backlight imaging [8,9], ending up with PDA (Phase Doppler Anemometry) [9] and LIF/Mie. Grout et al. [8] used Mie scattering to determine the size of individual droplets and calculate the evaporation rate according to D-square evaporation law. The droplets taken into account for diameter calculation covered at least several pixels what made possible to directly determine their diameters basing on Mie scattering images. This approach requires high magnification, what in turn limits the visualised area. Droplet size determination by means of backlight illumination is also based on individual droplets visualisation, and the same conditions need to be met (visualised droplet needs to cover at least several camera pixels). Therefore, in this case the visualised area becomes limited as well. In order to visualize individual droplets, Postrioti et al. [9] limited field of view of $2048 \times 2048$ pixel camera to $15 \times 15 \mathrm{~mm}$. This gave the scale factor of $7.3 \mu \mathrm{m} /$ pixel. In order to visualize the whole spray, they used backlight method. Then the field of view was of $100 \times 100 \mathrm{~mm}$. In that case however, the droplet size determination could not be made, and the results were used only to conclude on liquid mass distribution over the visualized area. Postrioti et al [9] used also PDA to determine droplet diameters. Due to the fact that PDA is a point measurement method, the spatial distribution required number of measurements. In their study the SMD was determined at 16 locations at distance of $90 \mathrm{~mm}$ and $140 \mathrm{~mm}$ from the injector outlet. They made 5 additional measurements for methods comparison which showed good accordance between the results obtained with PDA and backlight imaging. The 21 measurement locations done with PDA methods gave information only on part of the spray. In order to build the SMD distribution basing on PDA measurements the number of measurement points needs to be much higher. LIF/Mie technique can be an attractive alternative to discussed methods as it allows a rapid determination of droplet size distribution across the spray at the same time providing information on global spray parameters, specifically the spray angle. It has been successfully used for port fuel injection studies [10] where injection pressure is very similar to the one in SCR systems. The advantage of this method in reduced measuring times comes along with various challenging issues, such as accuracy limited by multiple-scattering, absorption effects and calibration procedures [10]. The calibration procedure is especially difficult for 90 deg detection angle since increase of the scattered light intensity with the droplet diameter is irregular [11]. Therefore, LIF/Mie ratio results shown in this study shall treated as qualitative indicator, not as direct SMD result. The multiple scattering effects are especially strong in case of optically dense sprays like non-evaporating diesel spray, where more than $65 \%$ of multiple light scattering is usually detected [12]. However, even in fairly dilute spray, where single scattering events are in majority, the conventional LIF/Mie technique still remains largely affected by errors introduced by multiple light scattering [13]. Structured Laser Illumination Planar Imaging (SLIPI) technique is an illumination and processing method which can remove signal coming from scattering at the droplets located outside illuminated area and therefore limits the errors caused by multiple scattering. It was developed for sprays by Berrocal et al. [14] and Kristensson et al. [15], and commercially implemented by LaVision [16]. The SLIPI concept is based on recording several modulated images where the modulation is vertically shifted between each recording [16]. Due to necessity of recording three images where sprays are illuminated with different modulation shift and the transient nature of spray, the image averaging is required. In high pressure conditions this causes setup complication since the flow conditions are required to scavenge the test chamber during the time between two consecutive injections [17]. Spray visualisation in ambient conditions doesn't pose any challenge since the recording can be usually repeated many times, and the averaging for each modulation phase can be done for high number of images. In this study, the injection process was observed in ambient conditions, therefore LIF/Mie ratio was determined using structured illumination. SLIPI LIF/Mie images were compared with conventional LIF/Mie images in order to determine if using structured illumination and SLIPI processing brings any benefit in such dilute sprays as SCR sprays.

The LIF/Mie experiments were supported by primary breakup visualization using shadowgraphy with long distance microscopy in order to determine relevant area for SMD calculation. Moreover, before main experiments the initial liquid jet velocity was measured using high speed imaging. The sprays were generated by a commercial automotive three-hole injector used in SCR systems. 


\section{Experimental setup}

The spray cloud created by three liquid jets emerging from the injector was illuminated using structured light sheet. In order to create modulated light sheet, the SLIPI optics delivered by LaVison was used. In the setup used in the study the three modulated images were used to create SLIPI image. The modulation shift was done by electric motor integrated into the SLIPI optics. In order to visualise whole spray cloud, the modulated light sheet in the area of interest was expanding in vertical direction. The schematic setup of the SLIPI optics is shown in Figure 1.

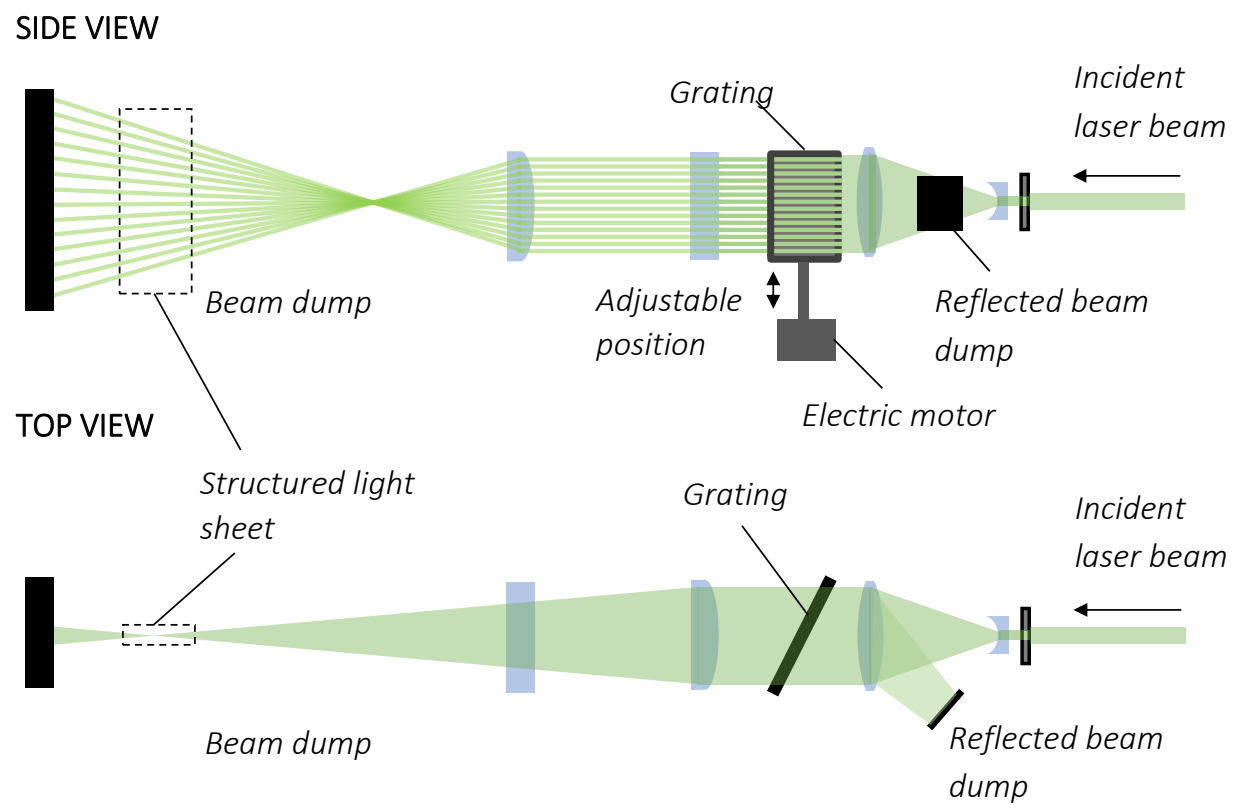

Figure 1. Schematic diagram of complete SLIPI optics.

Structured light sheet was created from second harmonic beam generated by Spectra-Physics Quanta-Ray Pro$230 \mathrm{Nd}$ :YAG $10 \mathrm{~Hz}$ pulse laser. The images were recorded by LaVision sCMOS cameras equipped with different filters. For Mie detection $532 \mathrm{~nm}$ bandpass filter was used, while for LIF $532 \mathrm{~nm}$ notch filter was applied. The shutter duration was adjusted to $15 \mu \mathrm{s}$ in both cameras. The schematic diagram of experimental setup is shown in Figure 2.

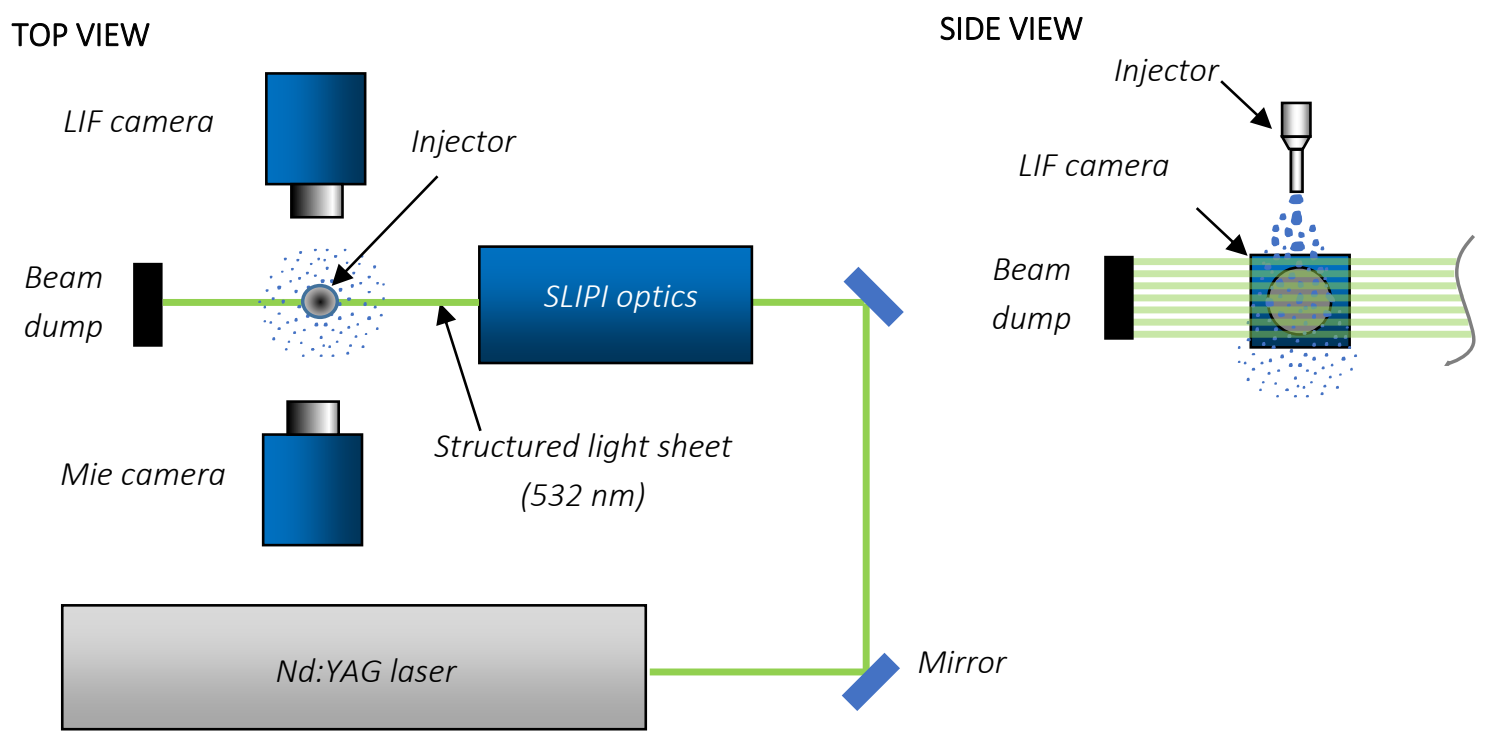

Figure 2. Schematic diagram of complete experimental setup. 
Because the modulated light sheet was expanding the period of modulation was dependent on the distance from the SLIPI optics. Therefore, the modulation period for calibration purposes was determined for the most relevant area - at distance of injector axis. The beam modulation period was calibrated using quartz cuvette filled with water doped with Eosin Y. The structured light sheet in the cuvette visualised with LIF and Mie cameras together with intensity profiles are shown in Figure 3 . Note that laser light sheet enters the visualisation area with $\mathrm{x}$ axis.
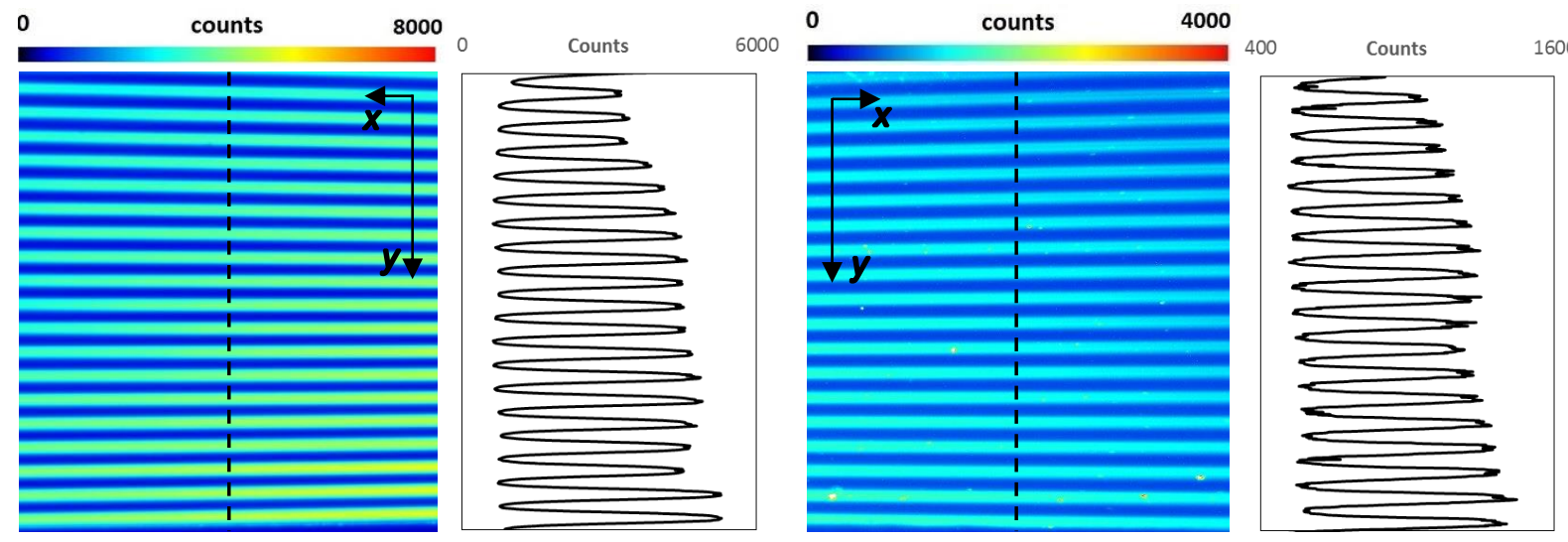

Figure 3. Structured light sheet used for calibration and the intensity profiles, LIF and Mie respectively from left.

The optics used in each camera was different, therefore the visible area in each camera was different as well. The system was calibrated in order to account for that difference and make possible to calculate LIF/Mie ratio. For this purpose, transparent plate with evenly distributed black dots was used.

Sprays were generated by a commercial 3-hole Bosch injector for SCR systems (no. 0280158 720). Instead of UWS pure water doped with Eosin $Y$ was used. Injection pressure was of 5 bar and the injection duration was of $15 \mathrm{~ms}$. The initial velocity of the liquid jet emerging the injector nozzle measured by high speed imaging at these conditions was of $25 \mathrm{~m} / \mathrm{s}$. During LIF and Mie recording the sprays were illuminated $10 \mathrm{~ms}$ after SOI (Start of Injection), when the sprays were fully developed. One LIF and one Mie image per injection was captured (for the same laser pulse). In order to specify the relevant area for LIF/Mie-based SMD calculation, the experiments were supported by shadowgraphy-based long distance microscopy measurements. Based on the microscopy results, the distance from injector outlet where no ligaments and strongly deformed droplets were present was determined. The setup for long distance microscopy is shown in [18].

\section{Results and discussion}

The image acquisition process for SLIPI visualisation consisted of capturing three series of images, each for different modulation phase, which could be then averaged. Berrocal et al. [12] recorded 100 images per each phase for diesel spray. According to the authors it was sufficient number for proper averaging. In this study 200 images were recorded for each phase. Capturing 200 images required 200 separate injections since only one image was captured per injection. The total number of recorded images for three modulation phases of the light sheet was of 600. One of two hundred instantaneous images and the averaged image for each modulation phase are shown in Figures 4 (LIF) and 5 (Mie). At each image the visualised area is of $90 \times 50 \mathrm{~mm}$. Both, instantaneous and averaged images are raw images without any processing and background subtraction. The injector axis is parallel to y axis.
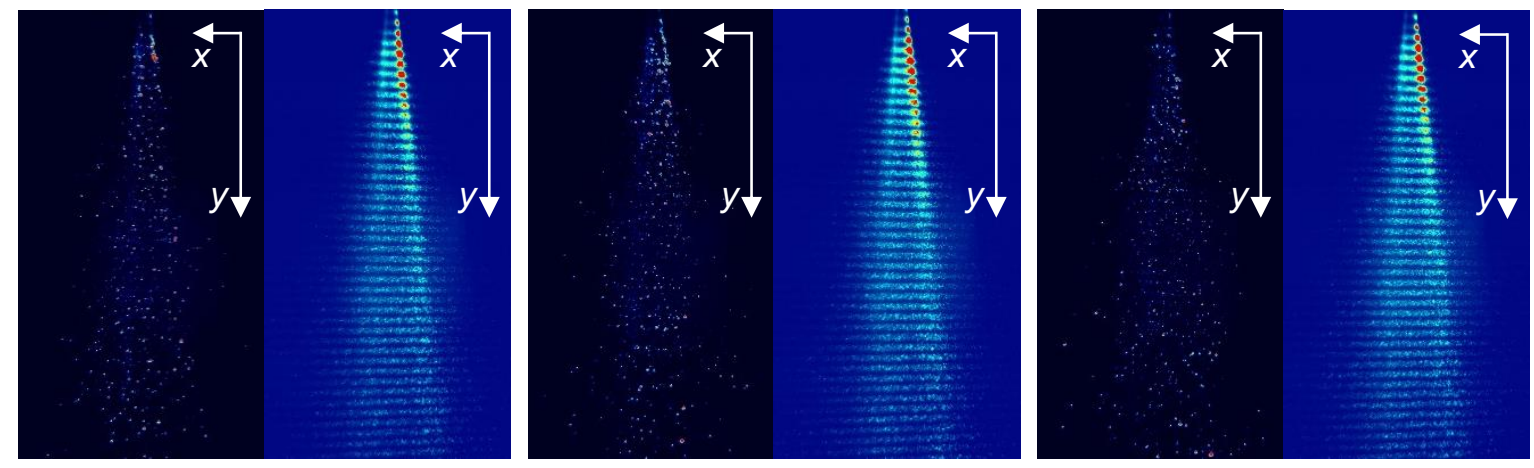

Figure 4. Instantaneous (left) and averaged (right) LIF images for each modulation phase; colour scale range 0-16000 counts (instantaneous images) and 0-8000 counts (averaged); the field of view at single image is of $90 \times 50 \mathrm{~mm}$. 

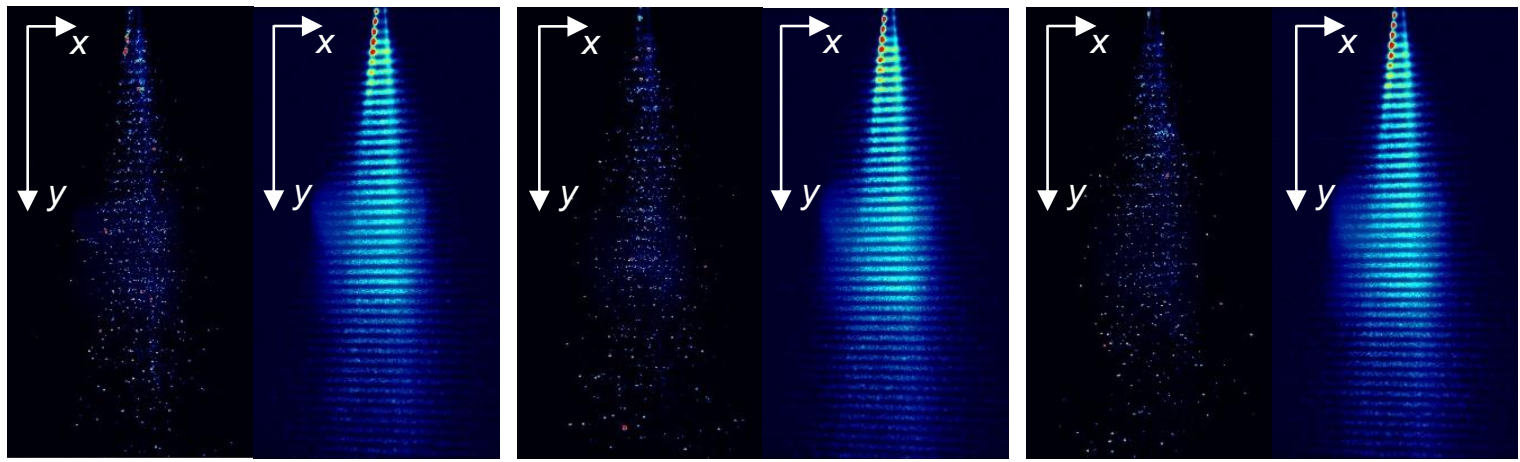

Figure 5. Instantaneous (left) and averaged (right) Mie images for each modulation phase; colour scale range 0-64000 counts (instantaneous images) and 0-16000 counts (averaged); the field of view at single image is of $90 \times 50 \mathrm{~mm}$.

Averaged LIF images exhibit relatively high background signal when compared to averaged Mie signal. Note that colour scale range is different for LIF and Mie images. Moreover, in case of LIF the background signal is uniform, while in case of Mie there is a clearly distinguishable area where it is strongly increased. This signal most probably comes from $532 \mathrm{~nm}$ notch filter placed at LIF camera, which reflects Mie signal scattered at droplets. That suggests that the arrangement based on dichroic mirror and location of both cameras at one side of the spray would be better solution in terms of background noise at raw images. This would be important when using conventional LIF/Mie but should not pose any challenge for SLIPI processing. The observed phase results shown in Figures 4 and 5 exhibit much stronger signal on one side of the spray, right and left respectively (note that the images are mirrored due to opposite locations of the cameras). This signal enhancement is not the effect of laser signal extinction as observed in single nozzle sprays $[12,17,19]$ but results from the fact that the spray cloud observed here is formed by three jets. The light sheet crosses one jet emerging from the injector (right side of the LIF image, and left side of Mie image) while going between two other jets.

Basing on three averaged modulated images (shown in Figures 4 and 5) conventional image was reconstructed (see Figure 6a). Strong background signal in the centre of Mie image was still present. The conventional images were processed to subtract the background signal (see Figure 6b). The background image was taken with laser pulse but without the spray. Although the overall background signal was substantially decreased, the signal in the centre of Mie image was still present. Therefore, additionally the measurement of Mie signal was done with masked LIF filter on the opposite camera. The average Mie image for masked LIF filter after background subtraction is shown in Figure 6c. After masking the filter the brighter region was not present, what confirms the assumption that the laser light was first scattered at the spray cloud and then reflected from the opposite camera filter.
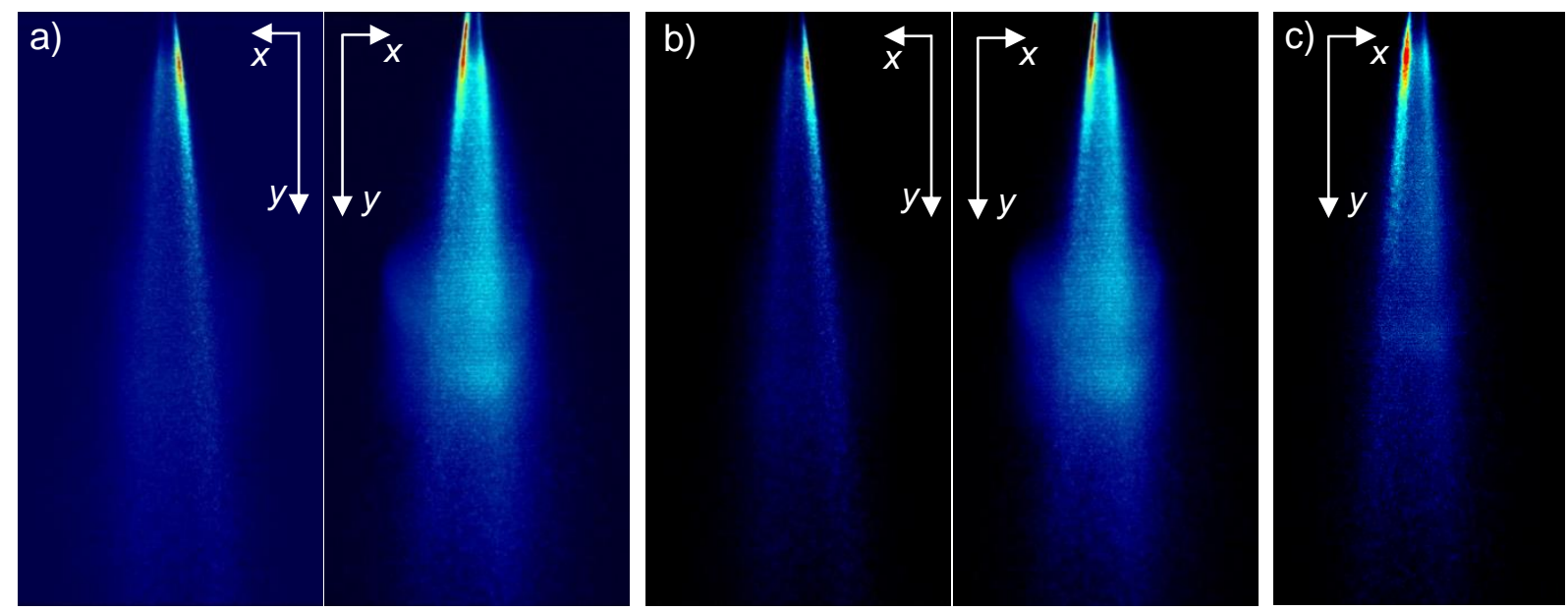

Figure 6. a) and b) Conventional LIF (left) and Mie (right) images reconstructed from three modulated averaged images; a) without background subtraction, b) with background subtraction c) Conventional Mie image obtained with masked filter on opposite camera (with background subtraction); colour scale range 0-8000 counts (LIF images) and 0-16000 counts (Mie images); the field of view at single image is of $90 \times 50 \mathrm{~mm}$.

The average phase images shown in Figures 4 and 5 were used to construct SLIPI image (shown in Figure 7a). Although Mie images shown in Figure 5 were affected by reflection from the filter they were selected for further analysis due to two reasons. The main reason was to construct LIF/Mie ratio basing on LIF and Mie signals from 
the same droplets. The other reason was to verify how SLIPI technique performs in removing this kind of reflections. Both, LIF and Mie SLIPI images were characterized by low background signal when compared to conventional image. The Mie SLIPI image appeared to be insensitive to recorded reflections from the camera filter, which were present at images used to build SLIPI image (averaged images shown in Figures 4 and 5).

SLIPI and conventional images were then used to calculate LIF/Mie ratio. The conventional images after background subtraction were taken for this analysis. The results are shown in Figure 7b. Note that LIF/Mie ratio was multiplied by 100 . LIF/Mie ratio distribution in case of conventional imaging was affected by discussed above camera reflections while SLIPI image was unaffected.
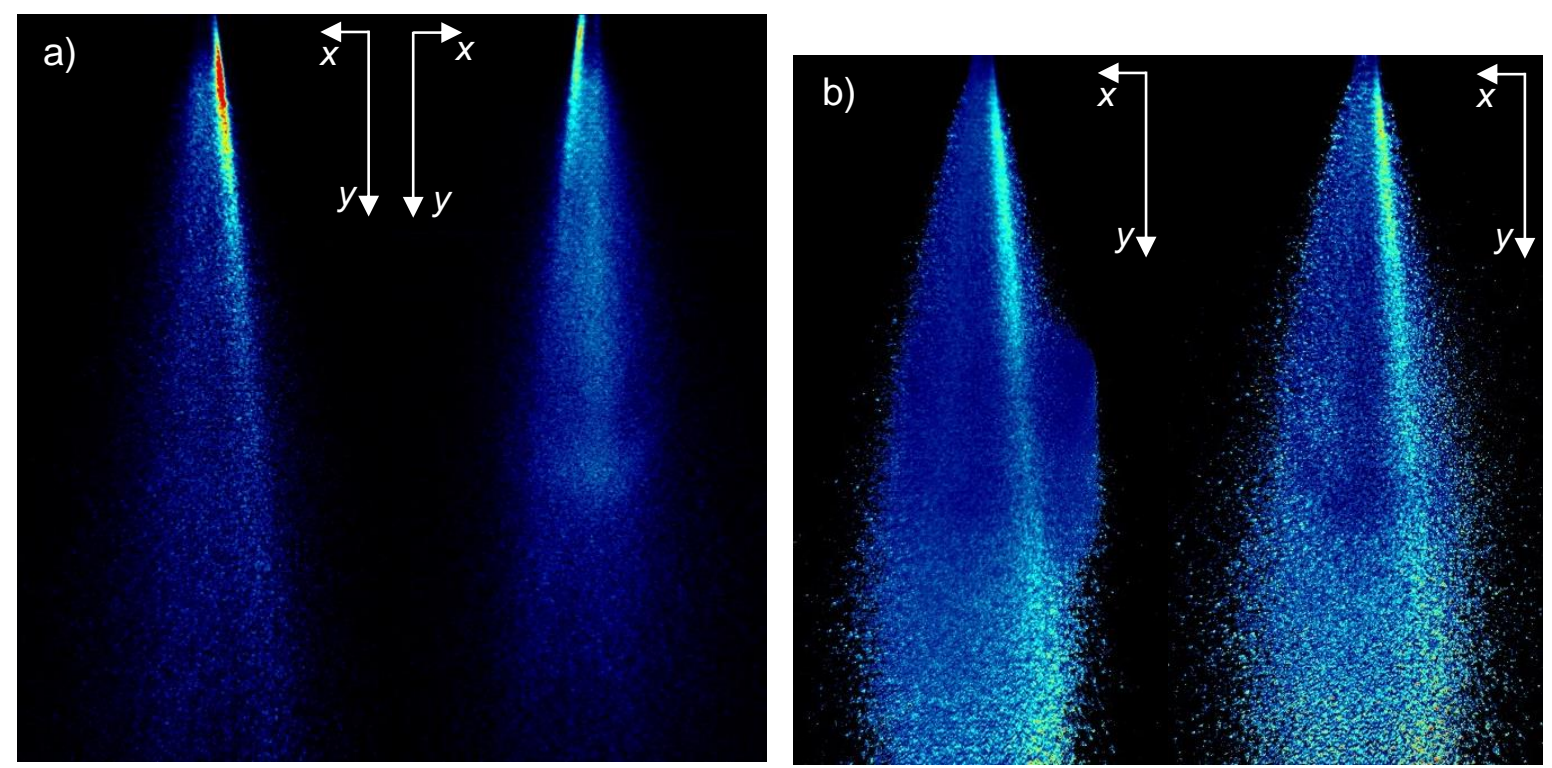

Figure 7. a) SLIPI LIF (left) and Mie (right) images, the field of view at single image is of $90 \times 50 \mathrm{~mm}$, colour scale range $0-4000$ counts (LIF) and 0-16000 counts (Mie). b) LIF/Mie ratio calculated from conventional images (after background subtraction) and from SLIPI images, respectively from left, the field of view at single image is of $85 \times 50 \mathrm{~mm}$, colour scale range $0-120$ counts.

In general, LIF/Mie ratio results are very similar for both type of imaging what indicates low multiple scattering effects. Nevertheless, the results obtained by SLIPI imaging seem to be more accurate. In case of conventional imaging LIF/Mie ratio decreases in the middle of the image. This observation suggests that the diameters of the droplets decrease in the middle of the visualised area to increase again at the end of the spray. This is questionable especially in case of the nozzle which was illuminated with the light sheet directly in the middle. In this case, it was expected to see either constant or gradually decreasing SMD along the nozzle hole axis. It needs to be considered that the LIF/Mie ratio could be affected by the non-spherical shape of droplets. Therefore, in order to determine how the LIF/Mie results could be affected by ligaments and strongly deformed droplets, the near-nozzle region was visualised with shadowgraphy-based long distance microscopy. The primary breakup visualisation showed that the unbroken liquid length oscillates between 5.2 and $6.4 \mathrm{~mm}$. Detached ligaments and strongly deformed droplets, however, were still observed further downstream up to around $11 \mathrm{~mm}$ from the injector outlet. The primary breakup and droplet formation is shown on two instantaneous images presented in Figure 8.

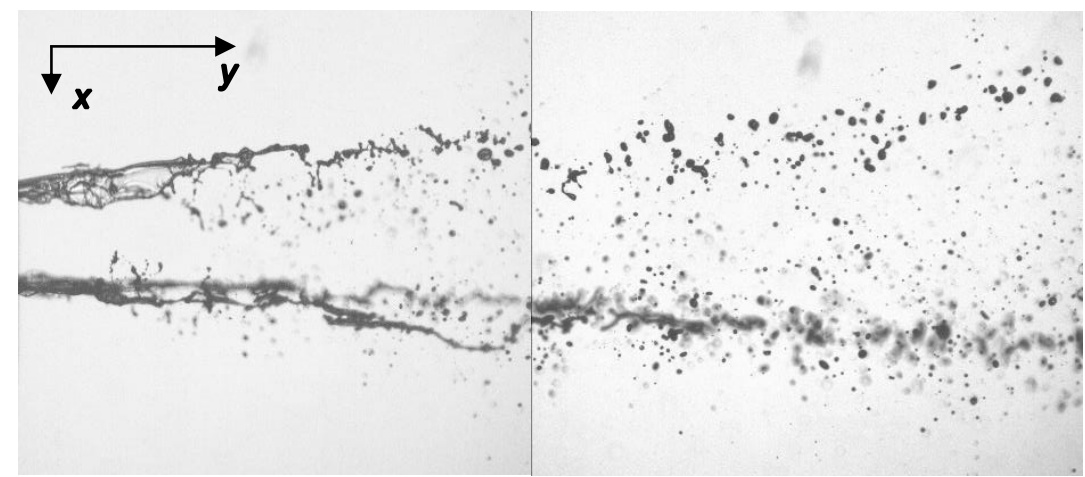

Figure 8. Long distance microscopy images - field of view $16.5 \times 7 \mathrm{~mm}$ (two images together); note that the images were taken during different injections. 
The injector position during shadowgraphy measurements was the same as during SLIPI recording. The images shown in Figure 8 were rotated by $90 \mathrm{deg}$ in order to connect them properly. Note that these images were taken during different injection events. The primary breakup experiments indicated that the area between the injector outlet and the distance of $11 \mathrm{~mm}$ from injector outlet is not relevant for LIF/Mie based SMD conclusions. In the area where ligaments were observed, typically high value of LIF/Mie ratio suggests high SMD. This area should be excluded from the analysis. Taking this into account one may notice that the LIF/Mie ratio does not change much with the distance from the injector. This is clearly visible in LIF/Mie ratio calculated from SLIPI images (shown in right image in Figure 7b). Constant LIF/Mie ratio suggests constant SMD. This in turn indicates that the secondary breakup does not play important role in the spray formation. This is in accordance to statement made by Birkhold et al. [20].

In both images shown in Figure $7 \mathrm{~b}$ LIF/Mie ratio is slightly increased at higher distance $(60-90 \mathrm{~mm})$. This can be related to the fact that bigger droplets are more prone to penetrate further, while the smaller ones due to lower momentum are decelerated easily by the aerodynamic forces. Lack of small droplets and the same size of large ones in that area causes the increase of SMD. This assumption however should be verified with PIV measurement. As stated above, the LIF/Mie-based droplet sizing is attractive since it can provide also information on global spray parameters, specifically the spray angle. Therefore, the conventional and SLIPI results obtained here were also used to determine the visualization angle of the entire spray cloud. For this purpose, only LIF images were used since conventional Mie images were affected by the reflections in background. Again, for this purpose the conventional images after background subtraction were used. The visualisation angle was determined assuming spray border as $90 \%$ of the recorded intensity. The visualisation angle determined from SLIPI image was of 18.06 deg while for conventional image was of 13.49 deg. $25 \%$ lower spray angle in case of conventional image suggests that conventional imaging performs relatively poor in the areas where signal is low. Then it is hardly distinguishable from the background.

\section{Conclusions}

Sprays emerging from the commercial automotive injector for SCR system were characterized by means of LIF/Mie method using structured illumination. SLIPI images were compared with conventional LIF/Mie images reconstructed from three modulated images. Additionally to LIF/Mie experiments near-nozzle area was visualized by means of shadowgraphy-based long distance microscopy in order to determine the area relevant for LIF/Mie-based conclusions on SMD. Ligaments and strongly deformed droplets were observed at distance of up to around $11 \mathrm{~mm}$ from the injector outlet indicating that the conclusions on SMD should not concern this area.

In general, LIF/Mie ratio results were very similar for both types of imaging, conventional and SLIPI. This indicates low multiple scattering effects. Moreover, the LIF/Mie ratio didn't change much with the distance from the injector. This suggests that the secondary breakup events are very rare. This is in accordance to statement made by Birkhold et al. [20].

Higher value of LIF/Mie ratio was observed at higher distance from the injector outlet $(60-90 \mathrm{~mm})$. This can be related to the fact that bigger droplets penetrate further while the smaller ones due to lower momentum are easily decelerated by the aerodynamic forces. Lack of small droplets and the same size of large ones (due to nonevaporating conditions) causes the increase of SMD. This assumption however should be verified with PIV measurement.

It needs to be noted that the conventional LIF/Mie images were affected by the incident reflections, coming from the camera filter located on the other side of the spray. This reflection was especially strong in Mie image.

Locating LIF and Mie cameras at one side of the laser light sheet and splitting LIF and Mie signals on dichroic mirror could be the solution for that. However, in certain cases it is not possible to get rid of background reflections. In case of SLIPI imaging the background signal didn't pose any challenge.

As far the experiments in the geometries resembling engine exhaust system are concerned this might be an important feature. Then the background reflections are expected to be present.

In the setup used in this study combination of these two approaches, conventional and structured illumination for LIF/Mie droplet sizing, could allow to conclude on the background signal origin and at the same time gave information on instantaneous droplet size distribution over the whole spray. In the setup used here the instantaneous LIF/Mie ratio is available only for conventional illumination since SLIPI used here requires three images obtained for different modulation phases of the structured light sheet. Interesting option for instantaneous imaging is two-pulse SLIPI based only on two phases as discussed by Payri et al. [21] and Kristensson et al. [22]. This approach however requires a dual cavity laser. This is planned to be done in the future.

In general, one can conclude that both methods, conventional and SLIPI, can be used for LIF/Mie ratio determination in such dilute sprays, provided that the sprays are visualised on non-reflecting background. SLIPI however should be used if a test rig generates any reflections. 


\section{Acknowledgements}

Current work was supported by the European Smart Growth Operational Programme 2014-2020 through the project "Development of mixing and urea-water solution conversion unit in SCR systems in order to start production of exhaust system for compression ignition engine that meets the Euro 7 emission standards", grant number: POIR.04.01.04-00-0060/15-02.

\section{References}

[1] Hirata, K., Masaki, N., Ueno, H., and Akagawa, H., 2005, "Development of Urea-SCR System for HeavyDuty Commercial Vehicles," SAE Tech. Pap. 2005-01-1860.

[2] European Comission, 2012, "Amendment No 459/2012 as Regards Emissions from Light Passenger and Commercial Vehicles (Euro 6)," Off. J. Eur. Union, 2012(459/2012), pp. 16-24.

[3] Jaworski, P., Kapusta, Ł. J., Jarosiński, S., Ziółkowski, A., Capetillo, A. C., and Grzywnowicz, R., 2015, "SCR Systems for NOx Reduction in Heavy Duty Vehicles," J. KONES, 22(4), pp. 139-146.

[4] Zheng, G., Fila, A., Kotrba, A., and Floyd, R., 2010, "Investigation of Urea Deposits in Urea SCR Systems for Medium and Heavy Duty Trucks," SAE Technical Paper, SAE International.

[5] European Commission, "Real-Driving Emissions in the EURO 6 Regulation on Emissions from Light Passenger and Commercial Vehicles (RDE3) - DRAFT REGULATION" [Online]. Available: https://ec.europa.eu/info/law/better-regulation/initiatives/ares-2016-6339064_en. [Accessed: 11-Jan2017].

[6] Kojima, H., Fischer, M., Haga, H., Ohya, N., Nishi, K., Mito, T., and Fukushi, N., 2015, "Next Generation All in One Close-Coupled Urea-SCR System," SAE Technical Paper, SAE International.

[7] Michelin, J., Nappez, P., Guilbaud, F., Hinterberger, C., Ottaviani, E., Gauthier, C., Maire, P., and Couturier, T., 2015, "Advanced Close Coupled SCR Compact Mixer Architecture," SAE Technical Paper, SAE International.

[8] Grout, S., Blaisot, J.-B., Pajot, K., and Osbat, G., 2013, "Experimental Investigation on the Injection of an Urea-water Solution in Hot Air Stream for the SCR Application: Evaporation and Spray/wall Interaction," Fuel, 106(x), pp. 166-177.

[9] Postrioti, L., Brizi, G., Ungaro, C., Mosser, M., and Bianconi, F., 2015, "A Methodology to Investigate the Behaviour of Urea-Water Sprays in High Temperature Air Flow for SCR," Fuel, 150(x), pp. 548-557.

[10] Bareiss, S., Fuhrmann, N., Dreizler, A., Bacher, H., Höffner, J., Weishäupl, R., and Kügler, D., 2013, "Planar Droplet Sizing for Characterization of Automotive Sprays in Port Fuel Injection Applications Using Commercial Fuel," Flow and Combustion in Advanced Gas Turbine Combustors, J. Janicka, A. Sadiki, M. Schäfer, and C. Heeger, eds., Springer Netherlands, Dordrecht, pp. 445-461.

[11] Charalampous, G., and Hardalupas, Y., 2011, "Numerical Evaluation of Droplet Sizing Based on the Ratio of Fluorescent and Scattered Light Intensities (LIF/Mie Technique).," Appl. Opt., 50(9), pp. 1197-1209.

[12] Berrocal, E., Kristensson, E., Hottenbach, P., Aldén, M., and Grünefeld, G., 2012, "Quantitative Imaging of a Non-Combusting Diesel Spray Using Structured Laser Illumination Planar Imaging," Appl. Phys. B, 109(4), pp. 683-694.

[13] Mishra, Y. N., Kristensson, E., and Berrocal, E., 2014, "Reliable LIF/Mie Droplet Sizing in Sprays Using Structured Laser Illumination Planar Imaging," Opt. Express, 22(4), p. 4480.

[14] Berrocal, E., Kristensson, E., Richter, M., Linne, M., and Aldén, M., 2008, "Application of Structured Illumination for Multiple Scattering Suppression in Planar Laser Imaging of Dense Sprays," Opt. Lett., 16(22), pp. 17870-17881.

[15] Kristensson, E., Berrocal, E., Richter, M., Pettersson, S., and Aldén, M., 2008, "High-Speed Structured Planar Laser Illumination for Contrast Improvement of Two-Phase Flow Images," Opt. Lett., 33(23), pp. 2752-2754.

[16] Leick, P., Grzeszik, R., Arndt, S., and Wissel, S., 2011, "Suppression of Multiple Scattering Using Structured Light Sheets - A First Assessment for Diesel and Gasoline Spray Visualization," Proceedings of 24th European Conference on Liquid Atomization and Spray System, Estoril, Portugal, pp. 1-10.

[17] Pastor, J., and Benajes, J., 2002, "Planar Laser-Induced Fluorescence Fuel Concentration Measurements in Isothermal Diesel Sprays," Opt. Express, 10(7), pp. 309-23.

[18] Kapusta, Ł. J., Jaworski, P., Teodorczyk, A., and Kowalski, J., 2015, "Laser Based Diagnostic System for Spray Measurements," J. KONES. Powertrain Transp., 22(3), pp. 91-98.

[19] Pastor, J. V, Payri, R., Salavert, J. M., and Manin, J., 2012, "Evaluation of Natural and Tracer Fluorescent Emission Methods for Droplet Size Measurements in a Diesel Spray," Int. J. Automot. Technol., 13(5), pp. 713-724.

[20] Birkhold, F., Meingast, U., Wassermann, P., and Deutschmann, O., 2006, "Analysis of the Injection of UreaWater-Solution for Automotive SCR DeNOx-Systems: Modeling of Two-Phase Flow and Spray/WallInteraction," SAE Technical Paper, SAE International.

[21] Payri, R., Gimeno, J., Martí, P., and Manin, J., 2012, "Fuel Concentration in Isothermal Diesel Sprays through Structured Planar Laser Imaging Measurements," Int. J. Heat Fluid Flow, 34, pp. 98-106.

[22] Kristensson, E., Berrocal, E., and Aldén, M., 2014, "Two-Pulse Structured Illumination Imaging.," Opt. Lett., 39(9), pp. 2584-7. 